\title{
Inguinal hernia repair in patients under and over 65 years of age: a district general hospital experience
}

\author{
A Musbahi, ${ }^{1}$ P Abdulhannan, ${ }^{2}$ O Nugud, ${ }^{1 *}$ T Garud ${ }^{1 *}$ \\ ${ }^{1}$ Friarage Hospital, Northallerton, United Kingdom \\ ${ }^{2}$ Royal Victoria Infirmary, Newcastle, United Kingdom \\ *Joint senior authors
}

Corresponding author, email: peshang.abdulhannan@nhs.net

Aim: The aim of this study is to compare outcomes of laparoscopic and open hernias in the over and under $65 \mathrm{~s}$ at a district general hospital.

Methods: Data were collected retrospectively on patients who underwent a unilateral inguinal hernia repair from 2012 to 2016. Only open mesh Lichtenstein repairs and laparoscopic transabdominal pre-peritoneal (TAPP) mesh inguinal hernia repairs were included. The dataset included patients' demographics and comorbidities, type of surgery (open vs. laparoscopic), presentation (elective vs. emergency), length of stay and postoperative complications

Results: 255 patients comprised the study cohort. $126(49 \%)$ patients were under 65 years and $129(51 \%)$ were over 65. Laparoscopic surgery was performed in 149 patients (58\%), while open technique was used in $106(42 \%)$. A higher proportion of patients over 65 underwent open surgery compared to patients under $65(55 \% \mathrm{vs.} 28 \%, p<0.001)$. Patients over 65 had a higher ASA score $(p=0.0158)$ and more comorbidities (COPD, DM, Anticoagulation) when compared to younger patients. The number of postop complications were $13(10 \%)$ in the over 65 s compared to $14(11 \%)$ in the under $65 \mathrm{~s}(p=0.94)$. There was no statistical difference in length of stay between the over and under 65 patients $(p=0.06)$.

Conclusions: Despite more comorbidities in the over $65 \mathrm{~s}$, this study shows that there is no significant difference in complication rates between laparoscopic and open inguinal hernia repair irrespective of age category. Selection bias for the type of repair and the potential for an alpha error mean larger studies are required to show equivalence.

\section{Introduction}

The elderly population is increasing and will expand the number of patients with frailty and comorbidities undergoing general surgical procedures. ${ }^{1}$ Inguinal hernia repair remains one of the most common surgical procedures with $27 \%$ males and $3 \%$ of females having or developing an inguinal hernia. ${ }^{2}$ Laparoscopic procedures are increasingly being employed for hernia repair, ${ }^{3}$ due to their associated benefits over open surgery in terms of reducing length of stay, expediting return to normal activity and reducing perioperative pain.

Initial studies that recruited few elderly patients demonstrated higher recurrence rates in laparoscopic hernias compared to open. ${ }^{4}$ Subsequent studies on similar cohorts showed largely equal outcomes with shorter recovery rates in laparoscopic surgery in the hands of an experienced surgeon. ${ }^{5}$ Data in the elderly cohorts have been limited by studies using small numbers. ${ }^{6-8}$ Some studies focused on patients over 80 years old, suggesting that it is safe to perform laparoscopic and open inguinal hernia repair in this group..$^{9,10}$

Against this background the study aimed to compare the outcomes of laparoscopic transabdominal pre-peritoneal (TAPP) and open hernia repairs in the over and under $65 \mathrm{~s}$.

\section{Methods and materials}

\section{Study design}

Data were collated retrospectively on patients who underwent a unilateral inguinal hernia repair from January 2012 to December 2016 from a prospectively maintained database. These were performed or supervised by three general surgeons at a single district general hospital, who had all performed over 300 hernia repairs each prior to 2012 . The choice of open vs. laparoscopic approach was based on the patient's preference, their associated comorbidities and the surgeon's experience. All repairs were done under general anaesthesia with the addition of local anaesthetic infiltration at the end of the open procedures.

Patients' demographics and comorbidities were obtained from case notes and electronic discharge letters. The dataset also included type of surgery (open vs. laparoscopic), presentation (elective vs. emergency), length of stay and postoperative complications. Patients were examined by a qualified doctor in the out-patient clinics 6-8 weeks postoperatively, and subsequently followed up via their records for up to 12 months. 
For the complication section, inguinodynia was defined according to the European Hernia Society guidelines as pain lasting 3 months or more postoperatively. ${ }^{11}$ Wound infection was defined by positive cultures from a wound swab after clinical diagnosis.

\section{Inclusion and exclusion criteria}

Patients were excluded if they had an inguinal hernia repair as part of another procedure such as small bowel resection. Bilateral and recurrent hernia repairs were also excluded. Only open mesh Lichtenstein repairs and laparoscopic mesh inguinal hernia repairs were included. Synthetic meshes were used for all the cases, and all laparoscopic repairs were done using transabdominal pre-peritoneal technique (TAPP).

\section{Statistical analysis}

Statistical analysis was performed using R studio v 3.4. Categorical data were summarised as counts and percentages. Patients were classified into two groups based on age $>65$ and $<65)$. Complications were compared in both groups.

Fisher's exact test was used to compare the proportion of complications across both groups to assess whether a statistically significant difference exists.

Binary logistic regression was performed to adjust for comorbidities and the type of operation to assess whether age category is an independent predictor of complications.

All hypothesis testing was performed at 0.05 significance level (two-tailed). $P$-value less than 0.05 was considered statistically significant.

Post-hoc power analysis was also performed to assess the power of study to detect an effect size similar to that reported in the current study. The main outcome was the categorical occurrence of complications. The sample size needed to achieve $80 \%$ power to detect such an effect size was also assessed for future studies.

\section{Results}

255 patients comprised the cohort. $126(49 \%)$ patients were under 65 years and $129(51 \%)$ were over 65 . Only 16 were females $(6 \%)$. Laparoscopic surgery was performed in 149 (91 under 65 vs 58 over 65 years) patients (58\%), while open technique was used in 106 (42\%) (35 under 65 vs 71 over 65 years) (Table 1$)$.

As summarised in Table 2, there was a statistically significant difference between both groups with respect to the type of operation. A higher proportion of patients over 65 underwent open surgery compared to patients under 65 ( $55 \%$ vs. $28 \%, p<0.001)$.

ASA score was significantly different between the two groups, where the proportion of older patients with scores 2 and 3 was higher compared to younger patients ( $p=0.0158)$.

There was no difference between the groups in number of patients with COPD and Type-2 diabetes.

The proportion of patients taking warfarin/anticoagulants was higher in older patients compared to younger patients $(p=0.0541)$. There were more patients over 65 with other comorbidities compared to patients under 65 (18.6\% vs. $7.14 \%, p=0.011$ ) (Table 3 ). The number of postoperative complications were $13(10 \%)$ in those older than 65 years compared to $14(11 \%)$ in those younger than 65 years $(p=0.94)$. There was no statistical difference in length of stay between the over and under 65 patients. There was no reported mortality in our study (Table 4) (Figure 1).

Results also show that there were no significant differences between both age groups with respect to the number of patients who suffered from complications.

Stratifying complications by type of surgery (laparoscopic vs. open) as well as age ( $<65$ vs. $>65)$ presented in Figure 2 shows the following:

\begin{tabular}{lccc} 
Table 1: Descriptive statistics & & \\
\hline & & Count & Percentage \\
\hline \multirow{2}{*}{ Age category } & $<65$ & 126 & 49 \\
& $>65$ & 129 & 51 \\
Presentation & Elective & 247 & 97 \\
& Emergency & 8 & 03 \\
Gender & Female & 16 & 06 \\
\multirow{3}{*}{ Type of surgery } & Male & 239 & 94 \\
& Laparoscopic & 149 & 58 \\
& Open & 106 & 42
\end{tabular}

\begin{tabular}{|c|c|c|c|c|c|c|}
\hline & & \multicolumn{2}{|c|}{$<65(n=126)$} & \multicolumn{2}{|c|}{$>65(n=129)$} & \multirow[t]{2}{*}{$P$-value } \\
\hline & & Count & Percentage & Count & Percentage & \\
\hline \multirow{2}{*}{ Presentation } & Elective & 125 & 99 & 122 & 95 & \multirow[t]{2}{*}{0.08} \\
\hline & Emergency & 1 & 1 & 7 & 5 & \\
\hline \multirow{2}{*}{ Sex } & Female & 5 & 04 & 11 & 9 & \multirow{2}{*}{0.214} \\
\hline & Male & 121 & 96 & 118 & 91 & \\
\hline \multirow{2}{*}{$\begin{array}{l}\text { Total } \\
\text { complications }\end{array}$} & No & 112 & 89 & 116 & 90 & \multirow{2}{*}{0.94} \\
\hline & Yes & 14 & 11 & 13 & 10 & \\
\hline \multirow[t]{2}{*}{ Type of surgery } & Laparoscopic & 91 & 72 & 58 & 45 & \multirow[t]{2}{*}{$<0.001$} \\
\hline & Open & 35 & 28 & 71 & 55 & \\
\hline \multirow{2}{*}{$\begin{array}{l}\text { Length of stay (average) } \\
\text { days }\end{array}$} & Laparoscopic & 1 & & 0.787 & & \multirow{2}{*}{0.06} \\
\hline & Open & 0.156 & & 1.493 & & \\
\hline
\end{tabular}

Statistical analysis was performed using Fisher's exact test of association $* P<0.05$ 


\begin{tabular}{|c|c|c|c|c|c|c|}
\hline \multirow[b]{2}{*}{ Comorbidity } & \multirow[b]{2}{*}{ Value } & \multicolumn{2}{|c|}{$<65$} & \multicolumn{2}{|c|}{$>65$} & \multirow[b]{2}{*}{$P$-value } \\
\hline & & Count & Percentage & Count & Percentage & \\
\hline \multirow{3}{*}{ ASA } & 1 & 72 & 57.14 & 62 & 48.06 & \multirow{3}{*}{0.0158} \\
\hline & 2 & 54 & 42.86 & 65 & 50.39 & \\
\hline & 3 & 0 & 0 & 2 & 1.55 & \\
\hline \multirow{3}{*}{ COPD } & No & 124 & 98.41 & 120 & 93.02 & \multirow{3}{*}{0.245} \\
\hline & Yes & 0 & 0 & 3 & 2.33 & \\
\hline & Missing & 2 & 1.59 & 6 & 4.65 & \\
\hline \multirow{3}{*}{ T2DM } & No & 126 & 100.00 & 121 & 93.80 & \multirow{3}{*}{0.071} \\
\hline & Yes & 0 & 0 & 5 & 3.88 & \\
\hline & Missing & 0 & 0 & 3 & 2.33 & \\
\hline \multirow{3}{*}{$\begin{array}{l}\text { Warfarin / } \\
\text { anticoagulants }\end{array}$} & No & 121 & 96.03 & 113 & 87.60 & \multirow{3}{*}{0.0541} \\
\hline & Yes & 3 & 2.38 & 11 & 8.53 & \\
\hline & Missing & 2 & 1.59 & 5 & 3.88 & \\
\hline \multirow{2}{*}{ Other comorbidities } & No & 117 & 92.86 & 105 & 81.40 & \multirow{2}{*}{0.011} \\
\hline & Yes & 9 & 7.14 & 24 & 18.60 & \\
\hline
\end{tabular}

Table 4: Incidence of various complications across age groups (12 months follow up)

\begin{tabular}{|c|c|c|c|c|c|c|}
\hline \multirow[t]{2}{*}{ Complication } & \multirow[t]{2}{*}{ Occurred } & \multicolumn{2}{|c|}{$<65$} & \multicolumn{2}{|c|}{$>65$} & \multirow[t]{2}{*}{$P$-value } \\
\hline & & $n$ & $\%$ & $n$ & $\%$ & \\
\hline \multirow{3}{*}{ Hematoma } & No & 125 & 99.21 & 126 & 97.67 & \multirow{3}{*}{1} \\
\hline & Yes & 1 & 0.79 & 1 & 0.78 & \\
\hline & Missing & 0 & 0 & 2 & 1.55 & \\
\hline \multirow{3}{*}{ Inguinodynia } & No & 118 & 93.65 & 123 & 95.35 & \multirow{3}{*}{0.22} \\
\hline & Yes & 8 & 6.35 & 3 & 2.33 & \\
\hline & Missing & 0 & 0 & 3 & 2.33 & \\
\hline \multirow{2}{*}{ Ischemic orchitis/atrophy } & No & 126 & 100.00 & 125 & 96.90 & \multirow{2}{*}{ N/A } \\
\hline & Missing & 0 & 0 & 4 & 3.10 & \\
\hline \multirow{3}{*}{ Other complications } & No & 124 & 98.41 & 122 & 94.57 & \multirow{3}{*}{0.679} \\
\hline & Yes & 2 & 1.59 & 4 & 3.10 & \\
\hline & Missing & 0 & 0 & 3 & 2.33 & \\
\hline \multirow{3}{*}{ Recurrence } & No & 121 & 96.03 & 121 & 93.80 & \multirow{3}{*}{1} \\
\hline & Yes & 4 & 3.17 & 4 & 3.10 & \\
\hline & Missing & 1 & 0.79 & 4 & 3.10 & \\
\hline \multirow{3}{*}{ Wound infection } & No & 124 & 98.41 & 124 & 96.12 & \multirow{3}{*}{1} \\
\hline & Yes & 2 & 1.59 & 2 & 1.55 & \\
\hline & Missing & 0 & 0 & 3 & 2.33 & \\
\hline \multirow{2}{*}{ Total } & No & 112 & 88.89 & 116 & 89.92 & \multirow{2}{*}{0.22} \\
\hline & Yes & 14 & 11.11 & 13 & 10.08 & \\
\hline
\end{tabular}

Statistical analysis was performed using Fisher's exact test

\section{Laparoscopic surgery}

Although the total number of complications trended higher in patients younger than 65 years (11 vs. 7 ), this difference was not statistically significant $(p>0.05)$.

Of all complications, inguinodynia, tended to be more common in younger patients compared to older patients (7 occurrences vs. 1 occurrence, respectively), however this difference was not statistically significant.

In addition, recurrences were reported more in older patients but again this was not statistically significant.

\section{Open surgery}

The total number of complications trended to be more common in patients older than 65 years $(6$ vs. 3$)$. Three recurrences occurred and were all in patients younger than 65 . These differences were not statistically significant.

Using binary logistic regression, the odds of inguinodynia in patients older than 65 years is 0.2 , the odds in patients younger than 65 years, though this was not statistically significant.

None of the remaining complications were significantly different across both groups after adjusting for surgery type and comorbidities (Table 5). 


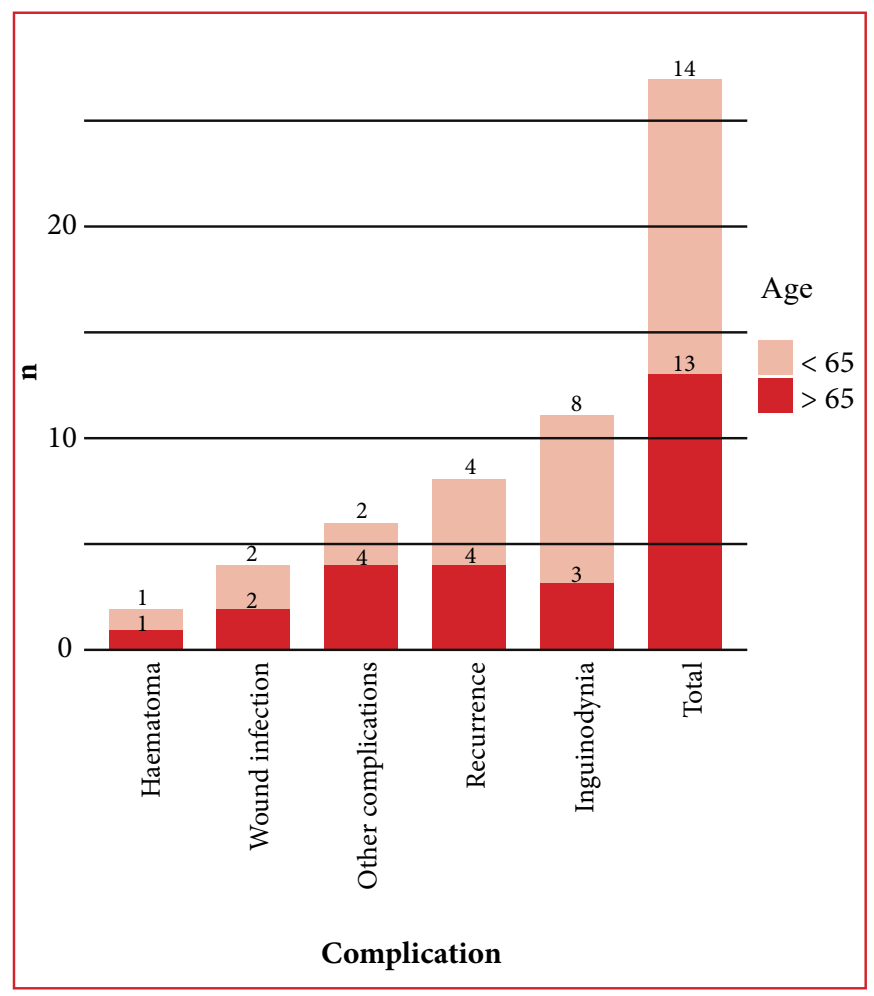

Figure 1: Complications for both laparoscopic and open surgery groups

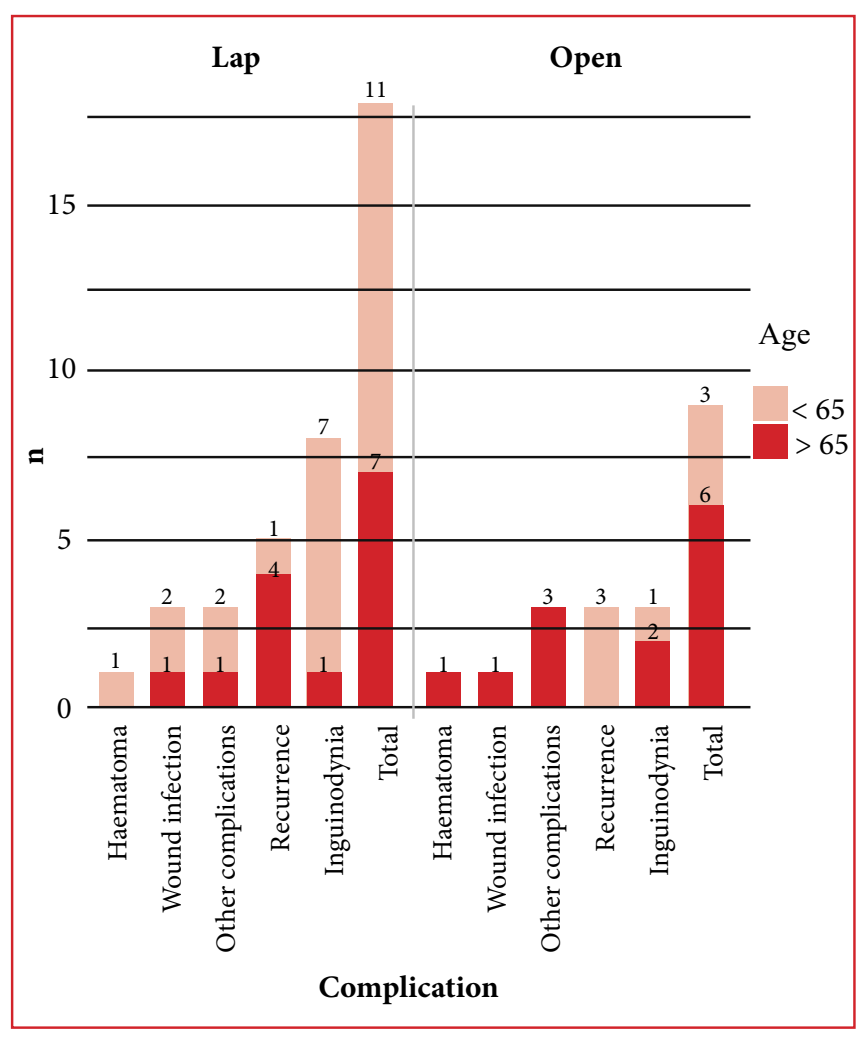

Figure 2: Complications by surgery type and age group

Table 5: Binary logistic regression to assess age as an independent predictor of various complications (12 months follow up)

\begin{tabular}{lccc}
\hline Complication & $\begin{array}{c}\text { Odds ratio for } \\
\text { Age }>\mathbf{6 5} / \mathbf{A g e}<\mathbf{6 5}\end{array}$ & $\mathbf{9 5 \%}$ CI & $P$-value \\
\hline Hematoma & 1.04 & $0.03-28$ & 0.928 \\
Inguinodynia & 0.22 & $0.04-0.927$ & 0.033 \\
Other complications & 1.28 & $0.198-10.5$ & 0.49 \\
Recurrence & 1.06 & $0.226-4.89$ & 0.99 \\
Wound infection & 0.809 & $0.08-7.79$ & 0.68 \\
Total & 0.797 & $0.326-1.905$ & 0.444
\end{tabular}

Results are adjusted for type of surgery, comorbidities. $95 \%$ CI that does not include one is statistically significant

Results show that the effect size (using total complications as the main outcome) in the current study was 0.034 (very small) and that the resultant study power (post-hoc power analysis) using the current sample size was 0.06 (very small). Further assessment showed that a sample size of 13910 patients (divided across both groups) is needed to provide $80 \%$ power to detect such an effect size.

\section{Discussion}

Laparoscopic surgery in the elderly places different physiological demands on the body than open surgery. ${ }^{12}$ Early studies reported mixed recurrence rates comparing laparoscopic and open repairs for all age groups., ${ }^{4,13}$ More recent studies pertaining to the elderly demonstrated comparable outcomes in elderly patients after laparoscopic and open hernia repairs, with some studies demonstrating no major morbidities and mortalities but an increase in postoperative seroma rate, urinary retention and re-admission. ${ }^{6,14}$ These comparable outcomes in more recent studies could be due to increased experience in laparoscopic inguinal hernia repair techniques.
In this small cohort over a period of 5 years, our study shows that there is no statistically significant difference in complication rates between laparoscopic and open inguinal hernia repairs in our setting. Our overall recurrence rate was $3.14 \%$ which is slightly higher than the average $0.5-2.2 \%$ recurrence rates reported in most large cohort studies. ${ }^{15,16}$ There is also no significant difference in recurrence rates in the over and under 65 populations, in both laparoscopic and open repairs, and no significant difference amongst all other outcomes. None of the patients in either group was diagnosed with ischemic orchitis/atrophy which is in keeping with the reported incidence of less than $0.2 \%$ in most of the literature.

Our results suggest that laparoscopic inguinal hernia repair is an equivalent operation in patients over the age of 65 when compared to open repair in our setting, and differences in outcomes were not detectable in a cohort of this size.

There is a noticeable heterogenicity of the definition of elderly in current clinical practice and research. ${ }^{17}$ There are no adequately age-adjusted studies of complications, although many large cohort studies suggested that age over 65 years is a risk factor for complications following inguinal hernia repair, especially in the presence of other factors such as bilateral or large inguinoscrotal hernias. ${ }^{16}$ There have 
also been some concerns regarding the physiological effects of pneumoperitoneum during laparoscopic surgery on the elderly patients. The use of $\mathrm{CO} 2$ leads to acid base and blood gas disturbances, while the increased intra-abdominal pressure leads to changes in pulmonary and cardiovascular physiology. ${ }^{18}$ However, there has been increasing evidence supporting the use of laparoscopic surgery in the elderly with the advantage of reduced postoperative pain, early mobility and shorter length of stay. ${ }^{19}$

Moreover, there is a difference in the preoperative performance status in both cohorts. Although, our data shows no difference in outcomes when adjusted for comorbidity, it is important to be vigilant in patient's selection and anaesthetic requirements for patients over the age of 65 .

Various studies suggested that preoperative assessment of patients and optimisation of their health prior to inguinal hernia repair may have a significant influence on positive surgical outcomes. ${ }^{20,21}$

Mayer et al. analysed a registry of 24571 patients who underwent laparoscopic inguinal hernia repair. They concluded that perioperative complication rates were higher in patients over 65 when compared to patients under $65 .{ }^{22}$ In 2018, the HerniaSurge group published their International Guidelines for groin hernia management. ${ }^{16}$ Their recommendations included the use of general instead of regional anaesthesia in patients older than 65 years to reduce complications associated with hernia repair, especially in the emergency setting. Similar recommendations were evident in earlier publications, although the use of local anaesthesia was also recommended as a preferred method in the elderly patients. ${ }^{23}$

Limitations of the study include the retrospective nature of it which meant that data had to be retrieved from case notes and operation systems that comes with the possibility of input error and missing data. Other limitations also included the fact that length of operation and return to normal activities were not compared between the groups, which could have been used as a quality marker in our cohort.

The use of local anaesthesia in open inguinal hernia repair has been found advantageous and was recommended by the European Hernia Society in 2009 as an alternative to general anaesthesia in patients with multiple comorbidities. ${ }^{11}$ It is considered preferable in patients with multiple comorbidities undergoing open inguinal hernia repair. ${ }^{16,24}$ None of the patients in our study had their hernia repaired under local anaesthesia which is another limitation of our study.

We were also unable to classify the hernias according to their sizes or types (direct/indirect). This was due to poor reporting by the surgeons in the operating notes, therefore this was considered as missing data.

BMI, preoperative level of physical activity and employment status could also be considered in this study. We did not record whether it was the trainee or consultant doing the operation, however, all operations were supervised by one of the three experienced consultant surgeons.

Quality of life tools could be used to assess short and long term satisfaction after laparoscopic or open hernia repair, although they require intense prospective follow up. Future research should also focus on the role of different laparoscopic techniques in the elderly patients.

\section{Conclusion}

Our study highlights that laparoscopic inguinal hernia repair (TAPP) in patients over the age of 65 is associated with comparable outcomes as open hernia repair in the same age group. It is, however, difficult to draw definitive conclusions based on this underpowered study. Larger studies with defined and comprehensive datasets are required to provide more meaningful results.

\section{REFERENCES}

1. Kurzer M, KarkA, Hussain ST. Day-case inguinal hernia repair in the elderly: a surgical priority. Hernia. 2009;13(2):131-6.

2. Bates AT, Divino C. Laparoscopic surgery in the elderly: a review of the literature. Aging and Disease. 2015;6(2):149.

3. Hamel MB, Henderson WG, Khuri SF, Daley J. Surgical outcomes for patients aged 80 and older: morbidity and mortality from major noncardiac surgery. J Am Geriatr Soc. 2005;53(3):424-9.

4. Neumayer L, Giobbie-Hurder A, Jonasson O, et al. Open mesh versus laparoscopic mesh repair of inguinal hernia. $\mathrm{N}$ Engl J Med. 2004;350(18):1819-27.

5. Takata MC, Duh QY. Laparoscopic inguinal hernia repair. Surg Clin. 2008;88(1):157-78.

6. Vigneswaran Y, Gitelis M, Lapin B et al. Elderly and octogenarian cohort: comparable outcomes with nonelderly cohort after open or laparoscopic inguinal hernia repairs. Surg. 2015;158(4):1137-44.

7. Ferrarese MD, Enrico S, Solej M, et al. Transabdominal preperitoneal mesh in inguinal hernia repair in elderly: end point of our experience. BMC Surg. 2013;13(2):S24.

8. Dallas KB, Froylich D, Choi JJ, et al. Laparoscopic versus open inguinal hernia repair in octogenarians: A follow up study. Geriatr Gerontol Int. 2013;13(2):329-33.

9. Pallati PK, Gupta PK, Bichala S, Gupta H, Fang X, Forse RA. Short-term outcomes of inguinal hernia repair in octogenarians and nonagenarians. Hernia. 2013;17(6):723-7.

10. Hope WW, Bools L, Menon A, Scott CM, Adams A, Hooks WB. Comparing laparoscopic and open inguinal hernia repair in octogenarians. Hernia 2013; 17(6):719-22.

11. Simons MP, Aufenacker T, Bay-Nielsen M, et al. European Hernia Society guidelines on the treatment of inguinal hernia in adult patients. Hernia. 2009;13(4):343-403.

12. Ceulemans R, Al-Ahdab N, Leroy J, et al. Safe laparoscopic surgery in the elderly. Am J Surg. 2004;187(3):323-7.

13. Liem MS, Van Vroonhoven TJ. Laparoscopic inguinal hernia repair. Br J Surg. 1996;83(9):1197-204.

14. Sivasankaran MV, Pham T, Divino CM. Incidence and risk factors for urinary retention following laparoscopic inguinal hernia repair. Am J Surg. 2014;207(2):288-92.

15. Kingsnorth A. Treating inguinal hernias: Open mesh Lichtenstein operation is preferred over laparoscopy. Br Med J. 2004;328(7431):59-60.

16. The HerniaSurge group. International guidelines for groin hernia management. Hernia. 2018;22(1):1-165.

17. Singh S. Defining 'elderly' in clinical practice guidelines for pharmacotherapy. Pharm Pract (Granada). 2014;12(4):489.

18. SafranD, Orlando R. Physiologic effects of pneumoperitoneum. Am J Surg. 1994;167(2):281-6.

19. Chesney T, Acuna SA. Do elderly patients have the most to gain from laparoscopic surgery? Ann Med Surg. 2015;4(3):321-3.

20. National Institute for Health and Care Excellence Guidelines. Available from: https://www.nice.org.uk/guidance/ng45/ evidence/full-guideline-pdf-87258149468

21. Köckerling F, Sheen AJ, Berrevoet F, et al. Accreditation and certification requirements for hernia centers and surgeons: the ACCESS project. Hernia. 2019;23(2):185-203.

22. Mayer F, Lechner M, Adolf D, et al. Is the age of $>65$ years a risk factor for endoscopic treatment of primary inguinal hernia? Analysis of 24,571 patients from the Herniamed Registry. Surg Endosc. 2016;30(1):296-306.

23. Hernia Interest Group South Africa. Inguinal hernia guidelines. S Afr J Surg. 2015;53(2):73-80.

24. Compagna R, Vigliotti G, Bianco T, et al. Local anesthesia for treatment of hernia in elder patients: Levobupicavaine or Bupivacaine? BMC Surg. 2013;13(2):S30. 